Title

\title{
A new scaling rule for context-dependent moral judgments.
}

\section{Authors}

Sandra Gomis-Pont ${ }^{1,2 *}$, Mari Carmen Navarro-Plaza ${ }^{1,2}$, Joaquín Navajas ${ }^{3}$, Rodrigo Quian Quiroga ${ }^{4}$, Salvador Sala ${ }^{1}$, and Luis M. Martínez ${ }^{1 *}$

\section{Affiliations}

${ }^{1}$ Institute of Neurosciences, Spanish National Research Council-Universidad Miguel Hernandez, Sant Joan d'Alacant, Alicante, Spain.

${ }^{2}$ Barcelonaßeta Brain Research Center, Pasqual Maragall Foundation, Barcelona, Catalonia, Spain.

${ }^{3}$ Laboratory of Neuroscience, Universidad Torcuato Di Tella. Buenos Aires, Argentina.

${ }^{4}$ Centre for Systems Neuroscience, University of Leicester, Leicester, UK.

*Correspondence to: I.martinez@umh.es and alexandra.gomis@gmail.com

\section{Abstract}

Moral judgments are typically explained by a combination of either deontological considerations about the nature of actions, or quantitative assessments of the consequences of those actions. These proposals, however, have serious limitations such as being insensitive to personal biases and global circumstances. This study presents an alternative approach based on comparative affective evaluations that modulate responses as more contextual information is presented to the choice set. We show that, when we make a moral decision, we do not simply judge the action and/or its consequences, we judge the protagonist performing the action embedded in a given set of circumstances and we normalize their behavior using the same gain control mechanism that operates in other sensory and motor domains. The explanatory power of this novel approach is broader than that provided by traditional paradigms and can be easily applied to more ecologically relevant scenarios. 


\section{One Sentence Summary}

Predictive affect-based gain control via divisive normalization governs contextdependent moral decisions.

\section{Main text}

From the role of attention in visual perception to the integration of concurrent multisensory information, and even the encoding of reward and value in economic decisions, the particulars of a wide range of different cognitive phenomena have been recently explained by a form of adaptive gain control called divisive normalization (1-6).

Divisive normalization is an operation that relies on comparative evaluations that modulate responses as more contextual information, which falls outside the response field, is presented or added to the choice set $(7,8)$. The success of divisive normalization in explaining even the most dramatic response changes reported in such different context-dependent behaviors has led to the proposal that it might function as a general brain computation, both in animals and humans $(8,9)$, perhaps with the striking exception of moral decisions $(10,11)$.

Current approaches to moral decision-making are strongly influenced by different versions of the dual process model (11-16): they propose that moral decisions are mostly based on a combination of either absolute normative considerations (deontology), judging only the nature of actions, or quantitative assessments of those actions' consequences (utilitarianism or consequentialism) (17-19); as if they could be gauged independently of the identity of the protagonists involved, our previous knowledge, interpersonal biases, context of the action and other circumstantial factors (20).

But if, on the contrary, we accept that moral decisions are context specific, then divisive normalization, as a canonical form of brain computation, could also play a role in moral behavior. If that was the case, what parameter, other than actions and their consequences, should then be normalized? In our everyday lives, the stimuli most likely 
to elicit an affective response is probably other people (21). We propose here that the context-dependent variable underlying moral judgments might be our subjective interpersonal biases and social stereotypes $(22,23)$. The idea is that we could be constantly comparing what the protagonists of moral dilemmas do in a given behavioral context with what we think they should do based on our previous knowledge and history of social interactions. These ongoing comparisons via divisive normalization should result in a dynamic, relative rather than absolute, representation of the affect we feel towards these social stereotypes, which should then, in turn, correlate with the probability to consider a given action morally acceptable in one context but not in another. If this hypothesis is correct, we should be able to accurately predict the moral decisions a person would make in a given scenario by simply asking them about their impression of the protagonist of the scene, how much they like or dislike them, without even having to inquire about the moral status of the situation at all.

To test our hypothesis, we have designed a novel experimental paradigm based on a series of quadripartite, nested moral dilemmas inspired in the well-known visual phenomenon of successive contrast, in which the appearance of a patch of light (the test field) is affected by previously viewed light fields (inducing fields) (Supplementary Materials and Methods, page 3). Each dilemma presents a real life story taken from Spanish news agencies, except for one that was adapted from the popular Heinz dilemma by Kohlberg (24). All dilemmas pose negative actions. The first trials expose the main facts (protagonists, actions and consequences), which were held constant throughout dilemmas. This first part of the quadripartite dilemmas always includes as little contextual information as possible, which was then sequentially introduced in the following three trials to generate "moral contours" of increasing strength in the direction of promoting moral acceptability. In essence, these contours create a discontinuity between what the protagonists actually do and our expectations of what we feel they should do. The magnitude of these contrasts is subjective, as they take place not only against the backdrop of other events occurring at the same time, the contextual 
102

information that we sequentially add to the dilemma, but also against the backdrop of the vast amount of information and social interactions that we have experienced through our lives and those that have been culturally inherited. If this is correct, moral contours should determine our personal affective evaluation of the protagonists of the moral scenes just like the luminance contrasts determine our responses to visual stimuli. Supplementary Text (Cronbach's Alpha Analysis, page 20) includes a psychometric analysis to demonstrate the inter-relatedness of the items across different trials.

Using this approach, we measured three different behavioral variables: 1) Stereotypic Affect (SA; Experimental Group 1) which is a basic reaction about the likeability of the active character of the dilemma using a 5-point Likert scale [1= low; $5=$ high SA ( $n=14$; mean age $28.38 \pm 4.50$ SD)]. 2) Perceived Affect (PA; Experimental Groups 2 and 3): participants judge the likeability of protagonists, not in isolation, but involved in different situational and behavioral contexts. In this case, participants were asked to evaluate the PA towards the protagonist after hearing every one of the four trials that comprised the quadripartite moral scenes. Subjects were instructed to respond, as fast as possible after hearing the trial, the following question: "How much do you like this character in this situation?" using a 1-5 point Likert scale [1= low; $5=$ high PA ( $n=442$; mean age $21.86 \pm 4.456$ SD)]. Finally, 3) Acceptability (Acc; Experimental Groups 2 and 4) measures whether the action depicted in the dilemma was deemed morally acceptable or not in each context $(n=442$; mean age $21.86 \pm 4.456$ SD; these participants were the same as before) (See Supplementary Materials and Methods).

We first examined to what extent SA could modulate moral acceptability. SA scores for each protagonist were averaged across participants (See Supplementary Data). We obtained the average moral acceptability for each trial of each dilemma (See Supplementary Data). The Spearman's correlation (Fig. 1C) between SA (participants of Group 1: $n=14$ ) and acceptability (participants of Group 2: $n=442)$ was rather weak $\left(r_{s}=\right.$ -0.1956; two sided; $\mathrm{Cl}=95 \% ; n=40$ (trials); $\mathrm{p}>0.05$ ), indicating that moral decisions, far 
129 from depending on the affective reaction evoked by the stereotype of the protagonist

130 (SA) only, could also be modulated by the contextual circumstances $(C)$ in which the 131 action is embedded. The scatter plot in Fig. 1A shows that, on the other hand, the 132 correlation between PA and acceptability (participants of Group 2: $n=442$ ) was very 133 strong (see also Fig. S1-S3), suggesting that PA and not SA has a large impact on the 134 probability to accept as moral a given situation $\left(r_{s}=0.9396\right.$; two sided; $\mathrm{Cl}=95 \% ; n=40$ 135 (trials); $p<0.0001$ ).
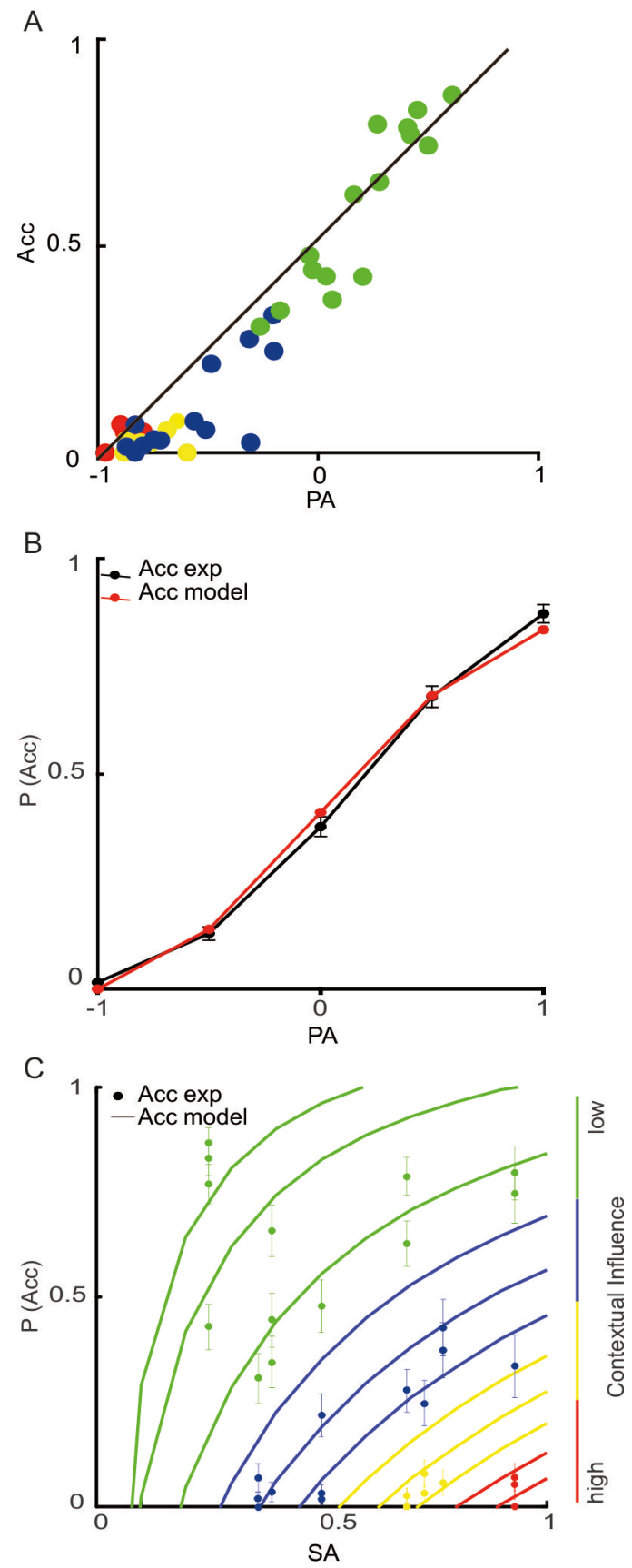
Fig. 1. Divisive normalization computation in moral decisions. A) Scatterplot showing the significant correlation $\left[r_{s}=0.9396\right.$; two sided; $\mathrm{Cl}=95 \% ; n=40$ (trials); $\left.p<0.0001\right]$ between average PA) and the acceptability (Acc) ratings of participants of Group $2(n=442)$. Colors indicate different levels of contextual influence. B) Prediction of the divisive normalization model. The red line represents experimental data [ $n=2572$ trials of participants of Group $2(n=442)]$ and the black line represents the modeled data obtained using the full divisive normalization equation. The constants' values were obtained using the Least Square method $[\beta=0.013 ; \gamma=0.8581 ; \mathrm{m}=1.987$; $\sigma=1.42 * 10 \mathrm{e}-7 ; \mathrm{SA}=1]$. Goodness of fit $\left[\mathrm{SSE}=0.0033 ; \mathrm{R}^{2}=0.9937 ; \mathrm{AIC}=-3.27^{*} 10 \mathrm{e} 4\right]$. The correlation between empirical and estimated data was statistically significant $\left[R^{2}=0.9951 ; n=5\right.$; $p<0.001]$. C) Dots represent the relationship between the acceptability (Acc) ratings (participants of Group $2(n=442))$ and the average SA (participants of Group $1(n=14))$ [n= 2572 trials] taking into account the weight of contextual influence (C). Different colored dots distinguish the different contextual conditions of the empiric al data. Colored lines represent the theoretical values of acceptability (Acc) modeled using the different contextual weights and the full divisive normalization equation. The constants' values were again obtained using the Least Square method $\left[\beta=1.1 ; \gamma=2.345 ; m=1 ; \sigma=9^{*} 10 e-3\right] ;$ goodness of fit $\left[S S E=0.2127 ; R^{2}=0.935 ; A I C=-\right.$ 203.4701]

We use a formalism originally described in visual perception to understand what computational representation mediates the effect of context on moral judgment. In this visual analogy, SA would be equivalent to the luminance of the central patch of a visual display, and the contextual influence to the luminance of the surround. Just like the appearance of the visual display, the PA towards the protagonist of the moral dilemmas would then be a function of the contrast between those two variables.

$$
P A=\frac{S A-C}{S A+C} \quad \text { equation } 1
$$

We then evaluated moral judgments in terms of affect-based divisive normalization (1-9). Such a context-dependent value representation of the protagonist of 
the dilemmas would be consistent with the known structure and function of most cortical circuits mediating sensory coding. The probability of accepting as moral a given action in a particular situation, $\mathrm{P}(\mathrm{Acc})$, depends on choice context, rescaling the driving input (SA) implementing a relative value (affect) code (1-9).

$$
P(A C C)=\gamma \frac{S A^{m}}{\sigma^{m}+\left(C^{m}+S A^{m}\right)}-\beta \quad \text { equation } 2
$$

The numerator includes the main driving input, and the denominator a constant $\sigma$ plus the normalization factor, which is the sum of the weight of the contextual information and the SA. The constants $\gamma, \sigma, \beta$ and $m$ are free parameters that are typically fit to empirical measurements: $\gamma$ determines overall responsiveness; $\sigma$ prevents division by zero and determines how responses saturate with increasing driving input, $m$ is an exponent that amplifies the individual inputs, and the constant $\beta$ sets the offset, simulating a baseline. The value of $C$ (contextual influence) is derived experimentally from the values of PA and SA using equation 1 (Supplementary Material and Methods). As predicted by the divisive normalization model of equation 2 , the probability to accept as moral a given situation depends linearly on the PA for the protagonist of the moral scene (Fig. 1A,B and Fig. S1) which in turn depends on a comparable evaluation between its SA and the contextual influence (Fig 1C). Values of PA higher than 0 led to a high probability of moral acceptance of the dilemma, while values below that score to a high probability of its rejection (Fig. 1B and Fig. S1 and Fig. S4).

Unlike our approach, current models of moral decision making put the focus on the type of dilemmas, actions and consequences; therefore, they tend to assume that people display the same character traits over time and in different situations. They even implicitly accept that if two individuals make the same behavioral decision is because they must share the same norm or belief about the situation. We found that, on the contrary, moral judgments depend more on individual differences in affect and context sensitivity than in the particulars of the dilemmas presented. Our quadripartite dilemmas, 
by always presenting negative actions with increasing weights of contextual information can be answered in 5 different ways that we call behavioral clusters (Supplementary Materials and Methods, page 15). Cluster D (for "Deontological") includes those subjects who have answered NO in the four trials of the dilemma; these are subjects who, in the case of that particular dilemma, have not been influenced by the context in which the action takes place. Cluster $\mathrm{C} 1$ represents those subjects who have answered YES from the first trial and have not changed their heart subsequently. These are participants who, from their own personal experience, have already internalized sufficient contextual information to dress up the dilemma and morally accept it from the very beginning. Clusters C2, C3, and C4 group individuals with different sensitivity or threshold to context. Our results in Fig. 2A show that there are dramatic and very characteristic differences in average PA between deontological (DR) and contextual responses (CR). Subjects in cluster D, present a modulation of PA in response to context that is never large enough to reach positive values and cross the acceptance threshold. C1 subjects, on the other hand, maintain equally large positive values of PA throughout the 4 trials of the dilemma. Finally, C2s, C3s, and C4s show similar profiles of perceived affect modulation but displaced to the right of each other as they require each more contextual information to cross threshold (25). Overall, moral acceptability thus seems to be a question of threshold to PA; the different contextual clusters show similar behaviors but in response to different parts of the dilemmas (Supplementary Text, page 22).

Furthermore, when we measure the acceptability profiles in a different group of subjects (Group 4: $n=35$; mean age $25.65 \pm 4.57$ SD; Supplementary Methods and Materials), the distribution of behavioral clusters is remarkably similar (Fig. S6), particularly if we take into account that they were obtained in two different countries (Spain and UK) and included different nationalities, cultural and religious backgrounds. 


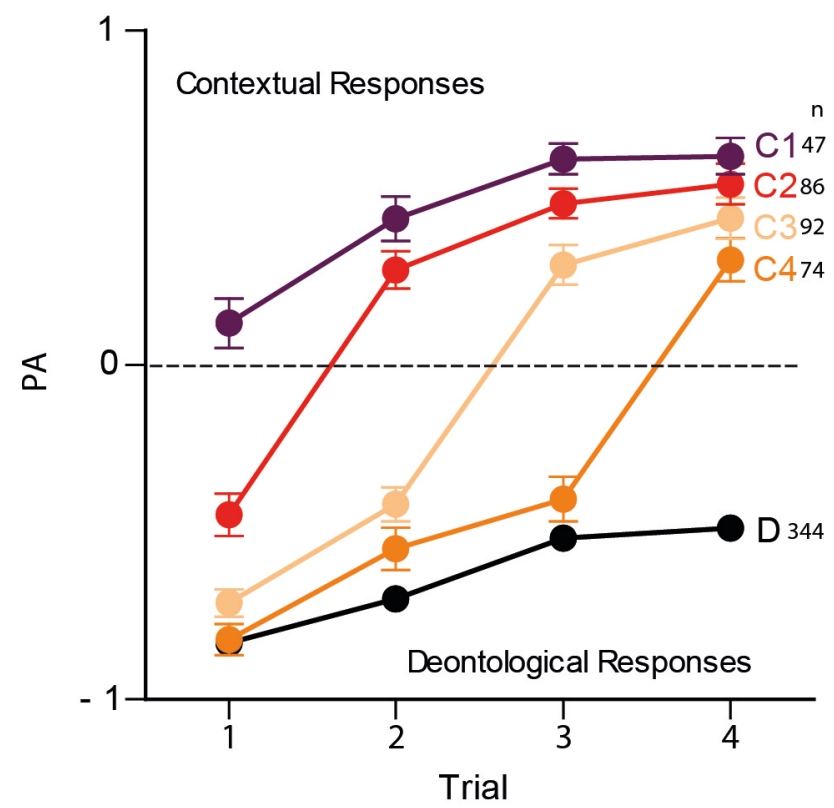

Fig. 2. Behavioral clusters and the different patterns of moral decisions. Relationship between PA and type of behavior in the four trials of the dilemmas (experimental group 2). Each point indicates the average PA ( \pm SEM) depending on the cluster and trial. Positive values of PA correspond to trials with contextual responses while negative values indicate trials with deontological responses. The dashed line at 0 represents the threshold for a contextual response. The sample's size of each cluster was: $D=344 ; C 4=74 ; C 3=92 ; C 2=86$; and $C 1=47$. A general Kruskal-Wallis test $(\mathrm{Cl}=95 \%$ : two sided $)$ was performed for each trial revealing statistically significant differences in PA scores between clusters [trial 1: $K=174.937, \mathrm{df}=4, \mathrm{n}=643$ trials, $\mathrm{p}<0.0001$; trial $2: \mathrm{K}=256.342, \mathrm{df}=4, \mathrm{n}=643$ trials, $\mathrm{p}<0.0001$; trial $3: \mathrm{K}=260.938, \mathrm{df}=4, \mathrm{n}=643$ trials, $p<0.0001$; and trail 4 : $K=283.268, d f=4, n=643$ trials, $p<0.0001]$. different timings and cognitive loads, are responsible for deontological and utilitarian, or contextual, responses $(14,18,26)$. In order to evaluate the cognitive load and timing associated to a moral decision, we measured the dynamics of the responses (Group 4: $\mathrm{n}=35$; mean age $25.65 \pm 4.57 \mathrm{SD}$; Supplementary Material and Methods) in one experimental group and found that our results also enter in contradiction with these 
tenets of the dual process model. First, in consonance with recent reports (27) we have found no latency differences (Wald $X^{2}=0.320$ : Two sided; $d f=1 ; C l=95 \% ; p=0.571$ ) between deontological (Mean= 2.955; $\mathrm{SEM}=0.101 ; \mathrm{n}=969$ (trials) group 4) and contextual trials (Mean= 3.257; SEM= 0.228; $\mathrm{n}=317$ (trials) group 4) (See Supplementary Text). Second, a Drift Diffusion Model, which assumes that decisions are made by a single noisy process that accumulates information over time from a starting point towards two criteria or boundaries, could accurately predict both the latency and proportion of contextual and deontological responses in our sample $(28)\left(X^{2}=1660000\right.$; Two sided; $d f=1 ; C l=95 \% ; p=0.24 ; n=1286$ (trials) group 4) (See Supplementary Text). Third, our results show that there are no statistically significant differences in cognitive load between deontological and contextual trials. Analyzing the dynamics of the choice process, we computed three parameters associated to the performance of each participant in response to each trial of each dilemma: a) Response time (RT) measures the time, or latency, elapsed between the end of the audio track and the mouse click on the response box; b) number of visual fixations, measured using the eye tracker (see Supplementary Materials and Methods, and movie S1), made by the subject while exploring the different elements of the response screen in the course of the response; and, c) the changes in acceleration experimented by the computer mouse while the participant moved it from the start box to the response box. We used these data to define both a swift and a slow trial per dilemma and subject. Swift trials were those with the lowest scores in at least two of the three parameters defined above (response time, visual fixation and mouse acceleration). Conversely, slow trials were those with the highest scores in at least two of those same three parameters (Supplementary Materials and Methods, page 26). 
A

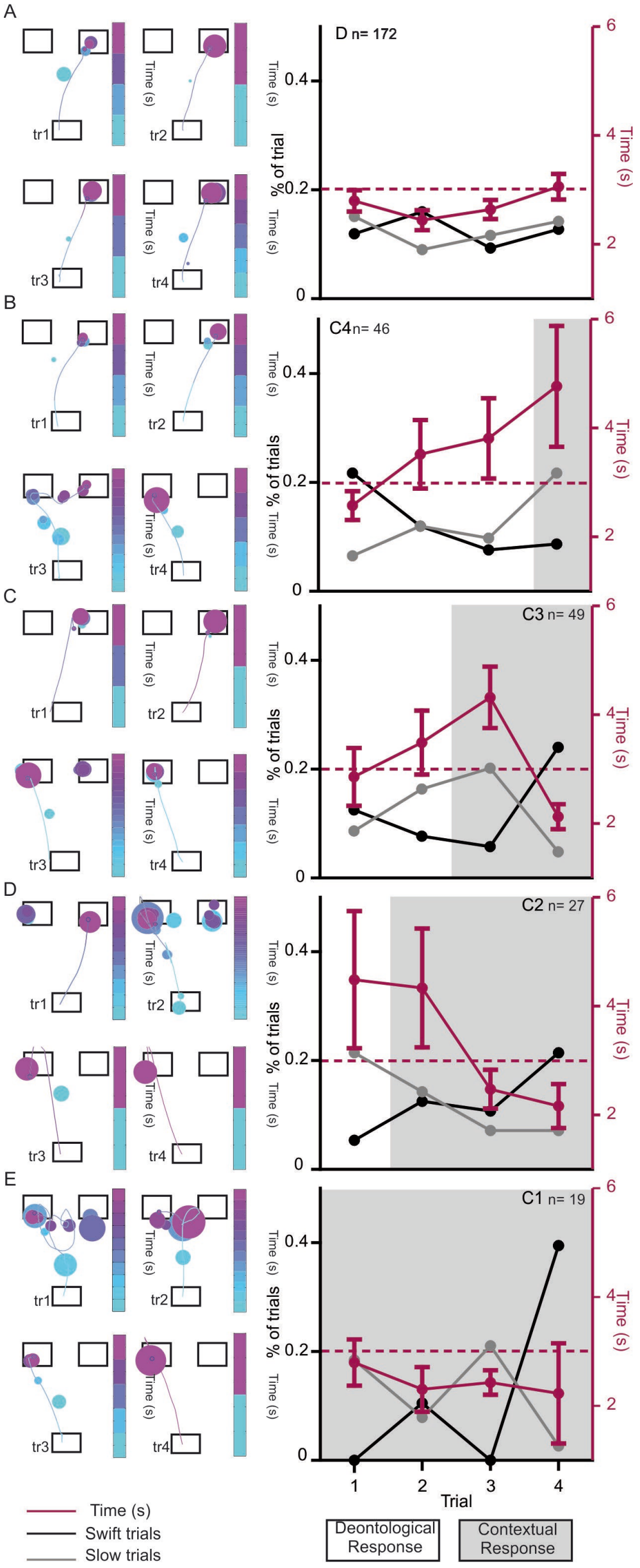


Fig. 3. Dynamics of the responses depending on the behavioral profile of the participants (group 4). A-E) Characteristic trial-by-trial evolution of the cognitive load profile associated to each behavioral cluster. The left column of the figure shows representative examples of each type of behavior. The starting box, at the bottom of the plot, and the response boxes, at the top, are shown as open rectangles. The colored lines trace mouse trajectories and the colored filled circles eye fixations, their diameters representing their durations. The different colors code for time intervals after audio offset (scale on the right). The line plots in the right column summarizes the different behaviors. The red lines represent the average response times (RTs) ( \pm SEM) to make deontological or contextual (shaded) decisions (right vertical axis). The black lines represent the proportion of trials associated with the lowest cognitive load (swift trials) and the gray lines represent the proportion of trials with the highest cognitive load (slow trials), both scaled in the left axis. Numbers on the upper corner of each graph represent the sample size of each cluster.

Our results show that there is a specific distribution of swift and slow trials characteristic of each type of behavioral cluster but not of each response type (Fig. 3), as it would have been predicted by dual process models. Deontological clusters (Fig. 3A) have no clear slow or swift trials. They are characterized by very fast responses (latencies were below 3 seconds, Fig. 3A right), few fixations and a straight mouse trajectory to the response box in every trial (Fig. 3A, left). C4 clusters (Fig. 3B) show a gradual increase in cognitive load as dilemmas progress and more contextual information is added. Trial 4 is usually the slowest and often classified as the slow trial; trial 1 is usually the fastest and swiftest one. C3 (Fig. 3C) and C2 (Fig. 3D) clusters, also show a gradual increase in cognitive load as the dilemmas proceed adding new context and up until the first contextual response. The last deontological and the first contextual response trials were the ones with the highest probability of being classified as slow, while the trials following the first contextual response were usually the swiftest ones. Average latencies also follow this pattern. In C1 clusters (Fig. 3E), the slow trial was often the first one in the run, while the swift trial was usually the last. Average response times were, as in the deontological clusters, below 3 seconds in every trial. Thus, our 
results reveal a clear dissociation between correlational analyses of response times and experimental manipulations of cognitive processes underlying moral judgments. Based only on cognitive loads or response times it would be impossible to accurately predict response type (deontological or contextual) (Supplementary Text), as dual process models defend $(14,18,26)$. This is what makes it so difficult that traditional single question paradigms can unambiguously shed light on the mechanisms that underlie our everyday life moral decisions. To assess whether with our nested, quadripartite paradigm we could predict both the dilemma that participants were responding to and the sign of their responses to each trial (the behavioral cluster to which they belonged in each case), we devised a doubt index (DI) based on different measures of the time that participants spent when making each moral decision (See Supplementary Material and Methods). Using a Linear Decoder, we were, in fact, able to predict above chance each subject's behavior simply by comparing the doubt index of trials 3 and 4 (Supplementary Text). Current models of moral judgment have been very influential and useful, but they largely underestimate the role that context plays in people's behavior (12-20). Here we show that when making a moral decision we do not simply judge the action and/or its consequences, we judge the protagonist performing the action embedded in a given set of circumstances and we normalize their behavior using the same gain control mechanisms that operate in other sensory and motor domains (1-9). This operation results in a subjective, and relative rather than absolute, representation of the PA towards the protagonist of the dilemma, which in turn accurately predicts both the latency and ratio of deontological and contextual responses.

PA thus emerges as the final common pathway of moral judgments, weighting the link of social relations and networks in which our behavior is embedded. In this regard, our model departs clearly from classic dual process models which propose that a fast, emotional, unconscious and automatic system is responsible for normative or deontological responses, while a slower and effortful reasoning process gives rise to contextual or utilitarian behaviors (12-20). To that extent, our model is more 
334

335

parsimonious and in tune with previous proposals suggesting that a single emotional system underlies moral judgment $(11,29)$, and with recent discoveries showing that complex behaviors have their basis in dynamic coalitions of networks of brain areas, none of which should be conceptualized as specifically emotional or essentially cognitive $(10,30,31)$.

When making moral judgments, people are strongly biased by personal and global circumstances (32). We are far more likely to consider and be affected by novel than familiar moral stimuli in general, a phenomenon akin to sensory and motor adaptation. Moral decisions are therefore also clearly experience-dependent, yielding what is subjectively considered the best possible behavior in light of available evidence (33). As in other domains, the strongest outputs are generated not necessarily by the stimuli of the highest absolute amplitudes, but by those imposing largest changes to the previous inputs; it is not all that uncommon to consider moral even the sacrifice of human lives and, at the same time, be inflexible with an allegedly minor offense such as a lie or a fraud. The mechanistic model we present here, based on the familiar divisive normalization operation, can account for this rapid rescaling of moral dynamic range and large deviations in moral behavior that we experience when social situations or circumstances change abruptly. Its explanatory power is, therefore, greater than that provided by classical approaches and it also has the advantage that it can be easily applied to more ecologically relevant scenarios.

4

5

(1)

\section{7}

8

59

\section{0}




\section{DATA AVAILABILITY}

The raw and source data are accessible in External Databases: the file with anonymized personal data of the experimental subjetcs, the scripts and the raw data of the eye- and mouse-tracking records are available in ready to use files on the open access repository of the CSIC (Spanish National Research Council), digital.csic.es, upon request during the review process and openly available afterwards under a link that will be provided.

\section{Acknowledgments}

We are grateful to Marcos Mirete and Sergio Molina for their help with some of the preliminary experiments. We would also like to thank Jorge Brotóns, Santiago Canals, Noemí Carranza, Felipe Criado-Boado, Karine Fauria, Álex Gómez-Marín, Carolina Minguillón, Eduardo Sánchez-Vila, Adrià Tauste, Arturo Valiño and members of the Institute of Neurosciences in Alicante for helpful comments and suggestions. This work was supported by the Spanish Ministry of Economy and Competitiveness (Grants BFU2010-22220 and BFU2014-58776-R to LMM), co-financed by the European Regional Development Fund (ERDF), and the Severo Ochoa Program for Centers of Excellence in R\&D (SEV-2013-0317). SG-P was a Fellow of the Spanish "Formación de Personal Investigador"-FPI program. 


\section{References.}

1. D. A. Ruff, M. R. Cohen, A normalization model suggests that attention changes the weighting of inputs between visual areas. Proc. Natl. Acad. Sci. U.S.A. 114(20), 4085-4094 (2017).

2. T. K. Sato, B. Haidor, M. Häusser, M. Carandini, An excitatory basis for divisive normalization in visual cortex. Nat Neurosci. 19, 568-570 (2016).

3. T. Ohshiro, D. E. Angelaki, G. C. DeAngelis, A neural signature of Divisive Normalization at the level of multisensory integration in primate cortex. Neuron. 95(2), 399-411 (2017).

4. K. Louie, L. E. Grattan, P. W. Glimcher, Reward value-based gain control: divisive normalization in parietal cortex. J. Neurosci. 31(29), 10627-10639 (2011).

5. K. Louie, T. LoFaro, R. Webb, P. W. Glimcher, Dynamic divisive normalization predicts time-varying value coding in decision-related circuits. J. Neurosci. 34(48), 16046-16057 (2014).

6. M. W. Khaw, P. W. Glimcher, K. Louie, Normalization value coding explains dynamic adaptation in the human valuation process. Proc. Natl. Acad. Sci. U.S.A. 114(48), 12696-12701 (2017).

7. V. Li, E. Michael, J. Balaguer, S.H. Castañón, C. Summerfield, Gain control explains the effect of distraction in human perceptual, cognitive, and economic decision making. Proc. Natl. Acad. Sci. U.S.A. 115(88), 8825-8834 (2018).

8. M. Carandini, J. D. Heeger, Normalization as a canonical neural computation. Nat Rev Neurosci. 13(1), 51-62 (2012). 
9. K. Louie, M. W. Khaw, P. W. Glimcher, Normalization is a general neural mechanism for context-dependent decision making. Proc. Natl. Acad. Sci. U.S.A. 110(15), 6139-6144 (2013).

10. M. J. Crockett, Models of morality. Trends Cogn. Sci. 17(8), 363-366 (2013).

11. F. Cushman, Action, Outcome, and Value: A Dual-System Framework for Morality. Pers Soc Psychol Rev 17(3) 273-92 (2013).

12. J. D. Greene, F. A. Cushman, L. E. Stewart, K. Lowenberg, L. E. Nystrom, J. D. Cohen, Pushing moral buttons: the interaction between personal force and intention in moral judgment. Cognition. 111(3), 364-371 (2009).

13. J. D. Greene, R. B. Sommerville, L. E. Nystrom, J. M. Darely, J. D. Cohen, An fMRI investigation of emotional engagement in moral judgment. Science. 293, 2105-2108 (2001).

14. J. D. Greene, J. M. Paxton, Moral reasoning: hints and allegations. Top Cogn Sci. 2(3), 511-527 (2010).

15. J. Schaich Borg, C. Hynes, J. Van Horn, S. Grafton, W. Sinnot-Armstrong, Consequences, action and intention as factors in moral judgments: an FMRI investigation. J Cogn Neurosci. 18, 803-817 (2008).

16. Shenhav, J. D. Greene, Integrative moral judgment: dissociating the roles of the amygdala and ventromedial prefrontal cortex. J. Neurosci. 34(13), 4741-4749.

17. M. Koenigs, L. Young, R. Adolphs, D. Tranel, F. Cushman, M. Hauser, A. Damasio, Damage to the prefrontal cortex increases utilitarian moral judgments. Nature. 446, 908-911 (2007). 
18. J. D. Greene, S. A. Morelli, K. Lowenberg, L. E. Nystrom, J. D. Cohen, Cognitive load interferes with utilitarian moral judgment. Cognition. 107, 1144-1154 (2008).

19. J. D. Greene, L. E. Nystrom, A. D. Engell, J. M. Darely, J. D. Cohen, The neural bases of cognitive conflict and control in moral judgment. Neuron. 44(2), 389-400 (2004).

20. J.D. Greene, "Fruit Flies of the Moral Mind" in What's Next: Dispatches from the Future of Science (Vintage, New York, 2009).

21. E.A. Phelps, K.M. Lempert, P. Sokol-Hessner, Emotion and decision making: multiple modulatory neural circuits. Annu Rev Neurosci. 37, 263-287 (2014).

22. D.M. Amodio, The neuroscience of prejudice and stereotyping. Nat Rev Neurosci. 15, 670-682 (2014).

23. M. Cikara, R. A. Farnsworth, L. T. Harris, S. T. Fiske, On the wrong side of the trolley track: neural correlates of relative social valuation. Soc Cogn Affect Neurosci. 5(4), 404-413 (2010).

24. L. Kohlberg, "The Development of Modes of Thinking and Choices in Years 10 to 16", thesis, University of Chicago, Chicago (1958).

25. M. Granovetter, Thresholds models of collective behavior. Am. J. Sociol. 83, 489515 (2014).

26. R. S. Suter, R. Hertwig, Time and moral judgment. Cognition. 119(3), 454-458 (2011).

27. J. McGuire, R. Langdon, M. Coltheart, C. Mackenzie, A reanalysis of the personal/impersonal distinction in moral psychology research. J. Exp. Soc. Psychol. 45(3), 577-580 (2009). 
28. R. Ratcliff, G. McKoon, The Diffusion Decision Model: Theory and Data for TwoChoice Decision Tasks. Neural Comput. 20(4), 873-922 (2008).

29. J. Haidt, The emotional dog and its rational tail: a social intuitionist approach to moral judgment. Psychol. Rev. 108(4), 814-834 (2001).

30. L. Pessoa, On the relationship between emotion and cognition. Nat Rev Neurosci. 9(2), 148-158 (2008).

31. H. Yu, J. Z. Siegel, M. J. Crockett, Modeling Morality in 3-D: Decision-Making, Judgment and Inference. Top Cogn Sci. Special Issue on Computational Approaches to Social Cognition,1-24 (2018).

32. J. Haidt, S. H. Koller, M.G. Dias, Affect, culture, and morality, or is it wrong to eat your dog? J Pers Soc Psychol. 65, 613-628 (1993).

33. P. S. Churchland, W. D. Casabeer, The Neural Mechanisms of Moral Cognition: A Multiple-Aspect Approach to Moral Judgment and Decision-Making. Biol. Philos. 18, 169-194 (2003).

34. L. De Rivera, M. R. Abuín, Listado de Síntomas Breves (LSB-50) (TEA Ediciones, Madrid, 2012).

35. L. R. Derogatis, Brief Symptom Inventory (BSY) (Pearson, San Antonio, 1992).

36. F. W. Cornelissen, E. M. Peters, J. Palmer, The Eyelink Toolbox: eye tracking with MATLAB and the Psychophysics Toolbox. Behav Res Methods Instrum Comput. 34(4), 613-617 (2002).

37. P. McCullagh, J. A. Nelder, Generalized Linear Models (Chapman and Hall/CRC Press, London, ed. 2, 1982). 
38. P. McCullagh, J. A. Nelder, Generalized Linear Models (Chapman and Hall/CRC Press, London, ed. 2, 1989).

39. M. Tavakol, R. Dennick, R. Making sense of Cronbach's alpha. Int J Med Educ. 2, 53-55 (2011).

40. R. Ratcliff, G. McKoon, The Diffusion Decision Model: Theory and Data for TwoChoice Decision Tasks. Neural Comput. 20(4), 873-922 (2008).

41. D. Wabersich, J. Vanderkerckhove, The RWiener Package: an R Package Providing Distribution Functions for the Wiener Diffusion Model. The $R$ Journal. 6(1), 49 (2014)

42. R. Bogacz, E. Brown, J. Moehlis, P. Holmes, J. Cohen, The physics of optimal decision making: a formal analysis of performance in two-alternative forcedchoice tasks. Psychol. Rev. 113(4): 700-765 (2006).

43. S. Verdonck, F. Tuerlinckx, Factoring out nondecision time in choice reaction time data: Theory and implications. Psychol. Rev. 123(2), 208-218 (2016).

44. J. Navajas, M. Ahmadi, R. Quian Quiroga, Uncovering the mechanisms of conscious face perception: a single-trial study of the $\mathrm{n} 170$ responses. J Neurosci. 33(4), 1337-1343 (2013).

45. R. Quian Quiroga, S. Panzeri, Extracting information from neuronal populations: information theory and decoding approaches. Nat Rev Neurosci. 10(3), 173-185 (2009). 
495 Supplementary Materials

496 Materials and Methods

497 Supplementary Text

$498 \quad$ Fig S1 - S6

499 Data S1 -S4

500 References (34-45)

501 Movie S1

502

503

504

505 